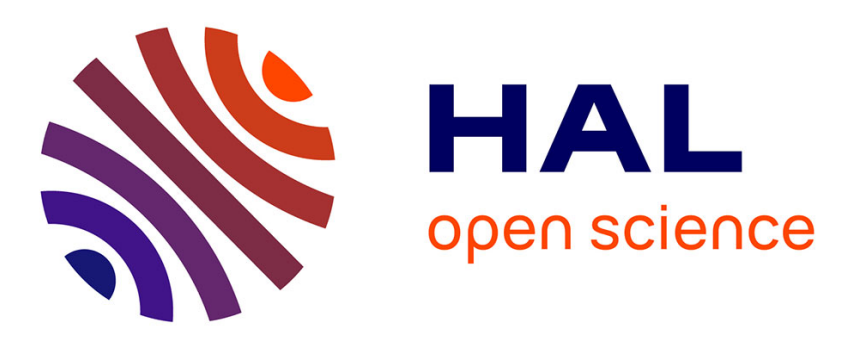

\title{
A novel method for measurement of dynamic light scattering phase function of particles utilizing laser-Doppler power density spectra
}

Stanislaw Wojtkiewicz, Adam Liebert, Hervé Rix, Piotr Sawosz, Roman Maniewski

\section{To cite this version:}

Stanislaw Wojtkiewicz, Adam Liebert, Hervé Rix, Piotr Sawosz, Roman Maniewski. A novel method for measurement of dynamic light scattering phase function of particles utilizing laser-Doppler power density spectra. Biomedical Optics and 3D Imaging OSA 2012, Apr 2012, Miami, United States. hal-00723247

\section{HAL Id: hal-00723247 \\ https://hal.science/hal-00723247}

Submitted on 8 Aug 2012

HAL is a multi-disciplinary open access archive for the deposit and dissemination of scientific research documents, whether they are published or not. The documents may come from teaching and research institutions in France or abroad, or from public or private research centers.
L'archive ouverte pluridisciplinaire HAL, est destinée au dépôt et à la diffusion de documents scientifiques de niveau recherche, publiés ou non, émanant des établissements d'enseignement et de recherche français ou étrangers, des laboratoires publics ou privés. 


\title{
A novel method for measurement of dynamic light scattering phase function of particles utilizing laser-Doppler power density spectra
}

\author{
S Wojtkiewicz ${ }^{1}$, Liebert ${ }^{1}$, H Rix $^{2}$, P Sawosz $^{1}$ R Maniewski $^{1}$ \\ ${ }^{1}$ Institute of Biocybernetics and Biomedical Engineering, Warsaw, Poland \\ ${ }^{2}$ Laboratoire d'Informatique, Signaux et Systèmes de Sophia Antipolis (I3S), UMR 6070 UNS/CNRS, Sophia Antipolis, France \\ Authore-mail address: swojtkiewicz@ibib.waw.pl
}

\begin{abstract}
We developed a novel method of measurement of the dynamic light scattering phase function of particles utilizing laser Doppler technique. We show the theoretical background, validation carried out on Monte Carlo data and proposition of measurement setup. OCIS codes: (290.5820) Scattering measurements; (170.3660) Light propagation in tissues, (170.5280) Photon migration, (170.3340) Laser Doppler velocimetry
\end{abstract}

\section{Introduction}

For medical applications of optical methods, knowledge of optical properties of biological tissues is an important issue. Parameters such as absorption coefficient, scattering coefficient and light scattering phase function determines light penetration path into the tissues. In this paper, we will focus on the light scattering phase function which is difficult to measure directly $[1,2]$. We developed a novel method of measurement of the light scattering phase function of moving particles utilizing laser Doppler (LD) power density spectra. In the clinical practice, the LD technique is used to assess tissues microperfusion [3]. We propose utilization of spectra measured by the LD instruments to evaluate the light scattering phase function of particles. Our method allows to measure phase function of particles of arbitrary shape in the full angular range, where conditions of the Mie scattering theory are fulfilled. This method could be applied especially in measurements of light scattering phase function of red blood cells.

We show the theoretical background of the method and its validation carried out using Monte Carlo simulations.

\section{Theory}

Doppler frequency change of the light wave during a single Doppler scattering event on a particle moving with speed $v$ is defined by the equation:

$$
\Delta f=\frac{2 n v}{\lambda} \sin \left(\frac{\theta}{2}\right) \cos \alpha,
$$

where $\alpha \in[-\pi ; \pi]$ is an angle between the moving particle velocity and a vector formed by subtraction of light vectors before and after scattering, $\theta \in[0 ; \pi]$ is the light scattering angle, $\lambda$ is the light wavelength and $n$ is the refractive index of the scattering medium.

Our goal is to estimate the distribution of angle $\theta$, which is a particular value of random variable $\Theta$. The probability distribution function (PDF) of $\Theta$ represents light scattering phase function of moving particles $f_{\Theta}(\theta)$. PDF of Doppler frequency shift $f_{U}(u)$ (where $\mathrm{u}$ is the frequency shift) is equal to the measured laser Doppler power density spectrum $S(\Delta f)$ normalized by its integral. The angle $\alpha$ is a particular value of random variable $A$. The PDF of $A-f_{A}(\alpha)$ is assumed to be known. For a particular measurement setup with known geometry, the probability distribution function of angle $\alpha$ can be calculated (e.g. using Monte Carlo simulation). The speed $v$ is a particular value of random variable $V$. The PDF of $V$ is assumed to be known and written as $f_{V}(v)$. In controlled environment, this assumption can be fulfilled.

Considering these assumptions, the equation (1) can be rewritten as follows:

$$
U=\frac{2 n}{\lambda} V \sin \left(\frac{\Theta}{2}\right) \cos A=C X Y=C Z
$$

where $C=2 n V / \lambda, X=\sin (\Theta / 2), Y=\cos A, Z=X Y$.

The LD spectrum represents a linear combination of Doppler shifts probability distributions for particular values of moving particles speeds $v$ [4-6]. Thus, the equation (2) can be represented by following Fredholm integral equation of the first kind:

$$
\mathbf{S}^{\mathrm{T}}=\mathbf{D} \mathbf{x}^{\mathrm{T}}
$$

where measured LD spectrum $\mathbf{S}$ : 


$$
\mathbf{S}^{1 \times N_{\mathrm{U}}}=\left[\begin{array}{llll}
f_{U}\left(u_{1}\right) & f_{U}\left(u_{2}\right) & \cdots & f_{U}\left(u_{N_{\mathrm{U}}}\right)
\end{array}\right],
$$

unknown vector $\mathbf{x}$, the PDF of random variable $X$ :

$$
\mathbf{x}^{1 \times N_{X}}=\left[\begin{array}{llll}
f_{X}\left(x_{1}\right) & f_{X}\left(x_{2}\right) & \cdots & f_{X}\left(x_{N_{X}}\right)
\end{array}\right]
$$

and coefficients matrix $\mathbf{D}$ :

where

$$
\mathbf{D}^{N_{\mathrm{U}} \times N_{\mathrm{X}}}=\left[\begin{array}{cccc}
\sum_{k=1}^{N_{\mathrm{C}}} d(1,1, k) & \sum_{k=1}^{N_{C}} d(1,2, k) & \ldots & \sum_{k=1}^{N_{C}} d\left(1, N_{\mathrm{X}}, k\right) \\
\sum_{k=1}^{N_{\mathrm{C}}} d(2,1, k) & \sum_{k=1}^{N_{\mathrm{C}}} d(2,2, k) & \ldots & \sum_{k=1}^{N_{\mathrm{C}}} d\left(2, N_{\mathrm{X}}, k\right) \\
\vdots & \vdots & \ddots & \vdots \\
\sum_{k=1}^{N_{\mathrm{C}}} d\left(N_{\mathrm{U}}, 1, k\right) & \sum_{k=1}^{N_{\mathrm{C}}} d\left(N_{\mathrm{U}}, 2, k\right) & \ldots & \sum_{k=1}^{N_{\mathrm{C}}} d\left(N_{\mathrm{U}}, N_{\mathrm{X}}, k\right)
\end{array}\right] .
$$

$$
d(i, j, k)=\left\{\begin{array}{lll}
\frac{1}{N_{\mathrm{X}} x_{j}} f_{V}\left(v_{k}\right) f_{Y}\left(\frac{u_{i}}{v_{k} x_{j}}\right) & \text { if } & u_{i} \in\left[0 ; v_{k}\right] \\
0 & \text { if } & \left.u_{i} \in\right] v_{k} ; u_{N_{\mathrm{U}}}
\end{array}\right]
$$

Two freeware Matlab toolboxes: Yalmip [5] and SeDuMi [6], utilizing the interior point method [7], were used to solve the problem (3).

Solution of the problem (3) is the PDF of random variable $X=\sin (\Theta / 2)$. Thus, the final form of the PDF of the light scattering angle $\theta$ can be represented by:

$$
f_{\Theta}(\theta)=\frac{1}{2} f_{X}\left(\sin \left(\frac{\theta}{2}\right)\right) \cos \left(\frac{\theta}{2}\right) .
$$

\section{Validation}

The method was validated with Monte Carlo (MC) calculated LD power density spectra. The code was adopted from previous studies $[4-6,10]$ and optimized for LD spectra simulations. MC simulations were carried out for a semi-infinite homogenous medium. Henyey-Greenstein phase function was used to simulate PDF of light scattering angle $\theta$ on moving particles Anisotropy factor $g$ defined as a mean value of cosine of the scattering angle $\theta$ was assumed $g=0.8$ and angle $\alpha$ is assumed to be uniformly distributed. Other simulation parameters are: scattering coefficient $\mu_{\mathrm{s}}=1 \mathrm{~mm}^{-1}$, absorption coefficient $\mu_{\mathrm{a}}=0.01 \mathrm{~mm}^{-1}$, refractive index $n=1.4$, laser light wavelength $\lambda=780 \mathrm{~nm}$, concentration of uniformly distributed moving particles $1 \%$, Gaussian moving particles speed distribution (mean speed $\bar{v}=2.786 \mathrm{~mm} / \mathrm{s}$ and standard deviation $\sigma=2 \mathrm{~mm} / \mathrm{s}$ ). LD power density spectra are sampled in the frequency range of $0-20 \mathrm{kHz}$. Photons are collected in a $0.2 \mathrm{~mm}$ wide ring-shaped concentric detector with the source-detector separation $r=1 \mathrm{~mm}$. The simulations were carried out for $30 \times 10^{8}$ photons.

In figure 1 the result of solving problem (3) utilized in equation (8) is compared with the Henyey-Greenstein phase function for assumed anisotropy factor $g=0.8$

Some discrepancies between both curves could be observed, which are mainly connected with the noise in the LD spectrum. Nevertheless, the anisotropy factors fit well. 


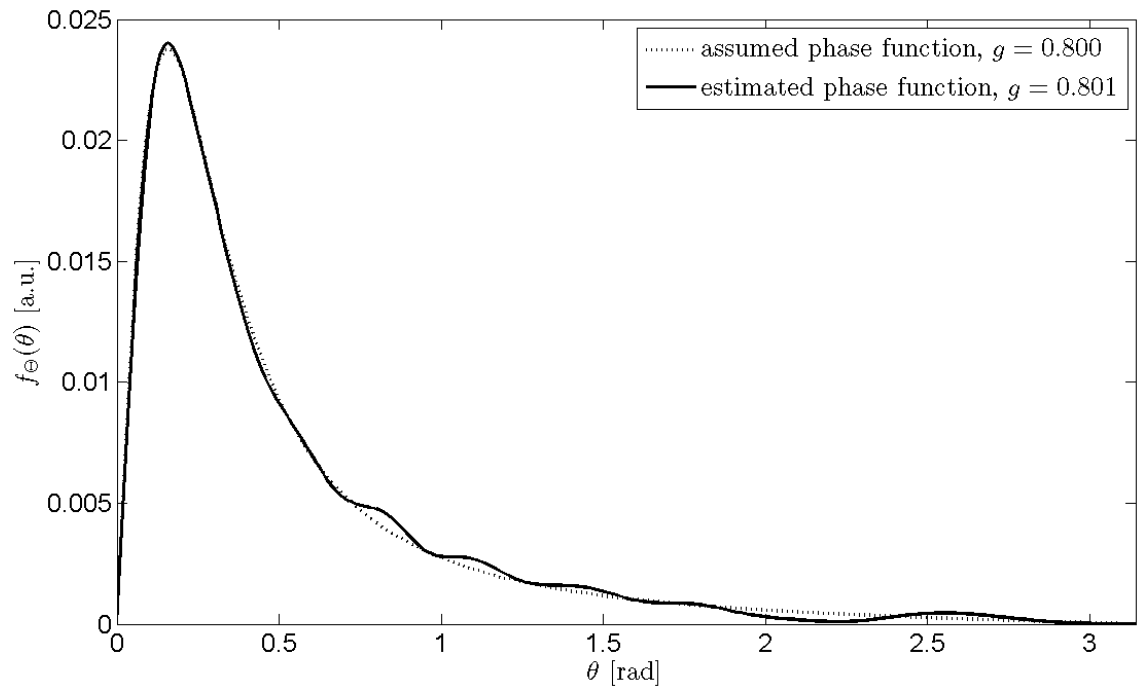

Figure 1. Comparison of assumed Henyey-Greenstein phase function for anisotropy factor $g=0.8$ and calculated phase function according to equations (3) and (8).

\section{Discussion and conclusions}

The proposed method could be utilized for measurement of light scattering phase function of moving particles. This measurement method and setup for assessment of light scattering phase function can be used for particles of any shape, where the Mie scattering theory could be applied. The setup could be made of glass tube (1mm diameter) fixed in an optically turbid material with the laser Doppler probe placed directly above the tube. Diluted suspension of the particles is pumped through the tube with known speed. Dilution of the particles eliminates influence of multiple Doppler scattering. The suspension flow through the tube should be laminar what means that suspension speed and tube diameter allows to calculate particles speed distribution. Optically turbid material surrounding the tube assures uniform distribution of angle $\alpha$.

Main limitation of the method is the necessity of dilution of the particles mixture. Nevertheless, this method potentially could be used in applications where knowledge of light scattering function of particles in the full angular range is required. The presented method is not limited only to the biomedical applications and could be applied for investigation of optical properties of wide range of nanoparticles.

\section{References}

[1] Martin Hammer, Dietrich Schweitzer, Bernhard Michel, Eike Thamm, and Achim Kolb, "Single Scattering by Red Blood Cells," Appl. Opt. 37, 7410-7418 (1998)

[2] M Hammer; A N Yaroslavsky; D Schweitzer 2001 A scattering phase function for blood with physiological haematocrit. Physics in medicine and biology 2001;46(3):N65-9.

[3] LeahyMJ et al 1999 Principles and practice of the laser-Doppler perfusion technique Technol. Health Care $7143-62$

[4] Liebert A et al 2006 Decomposition of a laser-Doppler spectrum for estimation of speed distribution of particles

moving in an optically turbid medium: Monte Carlo validation study Phys. Med. Biol. 51 5737-51

[5] Wojtkiewicz S et al 2009 Laser-Doppler spectrum decomposition applied for the estimation of speed distribution of particles moving in a multiple scattering medium Phys. Med. Biol. 54 679-97

[6] S Wojtkiewicz, A Liebert, H Rix and R Maniewski 2011 Evaluation of algorithms for microperfusion assessment by fast simulations of laser Doppler power spectral density Phys. Med. Biol. 56 (2011) 7709-7723

[7] L"ofberg J 2004 YALMIP: a toolbox for modelling and optimization in MATLAB Proc. CACSD Conf. (Taipei)

(http://control.ee.ethz.ch./joloef/yalmip.php)

[8] Sturm J F 1999 Using SeDuMi 1.02, a MATLAB toolbox for optimization over symmetric cones Optim. Methods Softw. 11-12 625-53

[9] Boyd S and Vandenberghe L 2006 Convex Optimization (Cambridge: Cambridge University Press)

[10] Liebert A, Wabnitz H, Steinbrink J, Obrig H, Moller M, Macdonald R, Villringer A and Rinneberg H 2004 Timeresolved multidistance nearinfrared spectroscopy of the adult head: intracerebral and extracerebral absorption changes from moments of distribution of times of flight of photons Appl. Opt. 43 3037-47 